Poster presentation

\title{
Flux balance analysis and its applications
} Balazs Papp* and Evangelos Simeonidis

\author{
Address: The University of Manchester, Manchester, UK \\ Email: Balazs Papp* - Balazs.Papp@manchester.ac.uk \\ * Corresponding author
}

from BioSysBio 2007: Systems Biology, Bioinformatics and Synthetic Biology

Manchester, UK. II-13 January 2007

Published: 8 May 2007

BMC Systems Biology 2007, I(SuppI I):P77 doi:10.1 I86/I752-0509-I-SI-P77

This abstract is available from: http://www.biomedcentral.com/I752-0509/I ?issue=S I

(c) 2007 Papp and Simeonidis; licensee BioMed Central Ltd.

The availability of annotated genome sequences has enabled the reconstruction of genome-scale metabolic networks for an increasing number of microorganisms. A popular and efficient method to study the characteristics and capabilities of such large-scale biochemical networks is flux balance analysis (FBA). In this tutorial we introduce the mathematical backgrounds of FBA and related methods, and present some of its recent biological applications ranging from biotechnology to evolutionary biology.

\section{Proposed content}

- Genome-scale metabolic reconstructions and their computational analyses.

- The constrained based approach to simulate large-scale models. Mathematical modelling and constrained optimisation. The basic idea of FBA.

- Using FBA to calculate growth properties and the effect of gene deletions in microbes. Experimental validation of computational predictions.

- Examples on the application of FBA in biotechnology.

- Application of FBA to understand genome evolution.

- Mathematics behind FBA: Linear Programming (LP) and Mixed Integer Linear Programming (MILP) formulations.

- LP solvers: the Simplex Method.
- MILP solvers.

- FBA as an LP problem.

- Mathematical examples. 